\title{
Aversive conditioning of naturally produced reward and nonreward odors in rats
}

\author{
W. ROBERT BATSELL, JR. and H. WAYNE LUDVIGSON \\ Texas Christian University, Fort Worth, Texas
}

\begin{abstract}
Past experiments have reported that rats encountering reward $(R)$ or nonreward (N) goal events emit odors that can be utilized as discriminative stimuli for instrumental behavior by conspecifics. In the present study, thirsty male rats were aversively conditioned by ingestional toxicosis to $R$ ard $N$ odors, and their suppression of water consumption in the presence of these odors was measured. Thirsty trained donors were placed into chambers containing $R$ or $N$ goal events to generate, respectively, the $\mathrm{R}$ or $\mathbf{N}$ odors. Test animals were given eight differential conditioning trials (four with one odor as CS+; four with the other as CS-), involving placement into an odorcontaining chamber with water available, followed by a $\mathrm{LiCl}$ injection on $\mathrm{CS}+$ trials. Animals tested in their CS+ odor consumed significantly less water than did CS- and control subjects. Both $\mathbf{R}$ and $\mathbf{N}$ odors were conditioned by aversive means and readily discriminable from each other. This represents the first laboratory demonstration of aversive conditioning of such naturally produced odors, and it suggests that aversive conditioning may be useful in the study of odorous emissions generally. Implications for innate meanings of $\mathbf{R}$ and $\mathbf{N}$ odors are discussed.
\end{abstract}

Numerous experiments have suggested that laboratory rats emit different odors into their environment during or in anticipation of goals of different value, such as reward $(\mathrm{R})$ and nonreward $(\mathrm{N})$, and that these odors are readily discriminated by other rats (e.g., Ludvigson, 1969; Ludvigson, Mathis, \& Choquette, 1985; Ludvigson \& Sytsma, 1967; McHose \& Ludvigson, 1966; Mellgren, Fouts, \& Martin, 1973; Morrison \& Ludvigson, 1970; Prytula \& Davis, 1974; Prytula, Davis, \& Fanning, 1981; Taylor \& Ludvigson, 1980). As a tactic of experimental efficiency, most studies have demonstrated discrimination by having odor arising from $\mathrm{N}$ (N odor) signal nonreward and odor arising from $R$ ( $R$ odor) signal reward. Eslinger and Ludvigson (1980) found that when odors were paired with "opposite," or incongruent, goal events the discriminative reaction was slightly delayed and somewhat smaller. Apparently, some natural predisposition retards the association of $\mathrm{N}$ odor with $\mathrm{R}$ consequences, and/or $\mathbf{R}$ odor with $\mathrm{N}$ consequences. In other words, there might be a certain biological "meaning" already associated with one or both of these odors.

With our growing understanding of the communication role of odorous emissions among animals, it is reasonable to suppose that $\mathbf{R}$ and $\mathbf{N}$ odors signal, or can readily come to signal, objects or events of significance to rats. As for the nature of what is signaled, the possibilities range from a specific reinforcer to a general class of reinforcers, with the class itself perhaps defined as much by the animal's characteristic reaction to the reinforcers as

We would like to thank Jennifer Peel for her technical assistance in data collection during this experiment. This research was supported in part by the Texas Christian University Research Fund. Reprint requests may be sent to W. R. Batsell or H. W. Ludvigson, Department of Psychology, Texas Christian University, Fort Worth, TX 76129 by their intrinsic properties. For example, certain emissions might signal to other rats the presence or absence of food (Voorhees \& Remley, 1981). At the other extreme, $\mathrm{N}$ odor might signal aversive events in general (including $\mathrm{N}$ ), and $\mathrm{R}$ odor would signal appetitive events in general (including $\mathbf{R}$ ). In either case, if information about reinforcing events is being conveyed by these odors, it would suggest an intriguing communication between animals that forage for their welfare. To understand any such communicative system, we must characterize the class of events signaled by an odor. The present work addresses this problem.

If the messages of these odors are general, an aversive conditioning paradigm might reveal it. Although there are many methods of aversive conditioning, ingestional toxicosis induced by lithium chloride $(\mathrm{LiCl})$ is an effective method for aversively conditioning an odor (Domjan, 1973). As part of the ingestional system, odors are readily conditioned, although not as readily as taste. Once associated with illness, they can alter ingestion and redirect exploratory behavior (Domjan, 1973). Past research in the area of aversive conditioning of odors has demonstrated that water consumption in the presence of an odor will be suppressed if that odor has previously been aversively conditioned (Domjan, 1973; Lorden, Kenfield, \& Braun, 1970; Panhuber, 1982; Taukulis, 1974).

In the present study, we placed rats into a chamber containing previously donated $\mathrm{R}$ or $\mathrm{N}$ odor, and gave them $\mathrm{LiCl}$ injections immediately upon removal from the odors. We then measured the extent to which the odor suppressed drinking. If the preexperimental meaning of these odors were rather general, animals aversively conditioned to $\mathrm{N}$ odor would be expected to show a greater suppression of water consumption than those aversively conditioned to $\mathrm{R}$ odor. This expectation assumes that $\mathrm{N}$ odor would 
be readily associated with aversive events because of its preexisting significance as a signal of such events, whereas $R$ odor's preexisting significance as a signal of appetitive events would provide no facilitation for, or might actually interfere with, learning the association between $R$ odor and aversive events.

In addition to the theoretical question, this study also addresses for the first time the basic empirical matter of whether these animal-produced odors can, in fact, be aversively conditioned. In so doing, it explores the usefulness of aversive differential conditioning as a technique for studying such odors.

\section{METHOD}

\section{Subjects}

Two groups of naive male rats from the Holtzman Company were used: 16 animals, approximately 200 days old $(500-600 \mathrm{~g})$ at the outset, served as donors of odors, and 60 rats, approximately 120 days old $(350 \mathrm{~g})$ at the outset, served as test subjects-either experimental or control. The animals were housed in standard Wahmann cages in the Texas Christian University vivarium, in which typical fluorescent lighting, present $24 \mathrm{~h}$ a day, was supplemented by natural daylight via windows.

\section{Apparauts}

The apparatuses were a straight runway and eight identical placement chambers. The straight runway was constructed of wood, painted white, and topped with a Plexiglas roof. It was $203 \mathrm{~cm}$ long, $7.62 \mathrm{~cm}$ wide, $8.89 \mathrm{~cm}$ high, and divided into three separate sections: a $30-\mathrm{cm}$ startbox, a $114-\mathrm{cm}$ run section, and a $59-\mathrm{cm}$ goalbox, with guillotine doors separating the sections. Time to traverse the runway was recorded by means of a microswitch on the door leading to the runway (start door) and photocells located 15, 136, and $166 \mathrm{~cm}$ from the start door. These provided start, run, and goal measures, respectively, although only the goal measure is reported here. A cylindrical stainless-steel drinking cup, $2.54 \mathrm{~cm}$ in diameter, $3.51 \mathrm{~cm}$ in height, and mounted on a $3.81 \times 8.89 \mathrm{~cm}$ base, was present on all trials in the runway, located just past the third photocell against the end wall of the goalbox.

Each placement chamber was $25.4 \mathrm{~cm}$ long, $7.62 \mathrm{~cm}$ wide, and $22.86 \mathrm{~cm}$ high. The side walls and one end wall of each chamber were constructed of plywood covered with formica; the second endwall was a piece of reinforced, cloudy Plexiglas. Chambers were covered with pieces of clear Plexiglas that could be lifted to allow placement and removal of a subject.

\section{Procedure}

All animals were fed Purina Rat Chow ad lib, but were on a waterdeprivation regimen of $40 \mathrm{ml}$ of water per day, beginning 4 weeks prior to test-subject conditioning. Water delivered in the home cage was presented in 50-ml calibrated polypropylene drinking tubes.

Donor conditioning took place concurrently in the straight runway and the experimental chambers. Meanwhile, the test subjects were gentled and handled, and their water intakes were recorded.

Donor conditioning. Fifteen donor animals were trained to run a runway in anticipation of liquid reward. (The 16th would not run in the runway, but was nonetheless used in placement chambers.) They were randomly assigned to two squads $(n=8$ and $n=7)$, which received 11 sessions of training before testing began. A training session involved eight trials in which either water $(R)$ or no water $(N)$ was given in the goal box in a double-alternation sequence, NNRRNNRR, which alternated with the reversed pattern, RRNNRRNN, every 2 days. In each trial, members of a squad ran consecutively in fixed order to the drinking cup. After one squad had completed a trial, the runway was swabbed with a damp cloth, then the second squad received a trial, before the first squad received its next trial, and so on, until all eight training trials were completed. The resulting intertrial interval was approximately $20 \mathrm{~min}$.

The donor rats also received 4 weeks of placement conditioning, with 30 daily sessions. The 11 training sessions in the runway apparatus coincided with the last 12 training sessions in the placement chambers. A daily session in the placement chambers consisted of eight placements for each donor. A typical placement involved a squad of 8 donors placed in the eight chambers for a period of $2 \mathrm{~min}$, with a drinking cup present. Following this period, each of the 8 donors in the second squad was placed into a chamber for $2 \mathrm{~min}$. After the removal of this second squad, all placement chambers were cleaned, and another placement trial was performed. On half of these trials, $10 \mathrm{ml}$ of water were in the cup, and on the other occasions, the cup was empty. The sequence of these $\mathbf{R}$ and $\mathbf{N}$ events was like that used in the runway. The rats received training in each of the eight placement chambers during the eight placement trials in a daily session.

Odor aversion conditioning. The test subjects were divided into six separate groups, equated for body weight and water intake. Body weight was the average over 2 days before conditioning, and water intake was the average daily consumption in the 4-week period before conditioning. The six groups were designated as (1) NN, poison nonreward/test nonreward; (2) NR, poison nonreward/test reward; (3) RR, poison reward/test reward; (4) RN, poison reward/test nonreward; (5) $\mathrm{CR}$, noncontingent control/test reward; (6) $\mathrm{CN}$, noncontingent control/test nonreward. One odor, $\mathrm{R}$ or $\mathrm{N}$, was designated as CS+ and aversively conditioned, with the alternate odor designated as CS- and not aversively conditioned. The first letter in an experimental group's label indicates which odor served as $\mathrm{CS}+$, while the second letter indicates the test odor. The CS+ and the CS- trials were given on alternate days, one trial per day for 8 days. Within each group, half of the subjects received their CS+ odor on the first day, while the other half received their CS- odor.

Each test subject was assigned a particular pair of donors that always provided its odors. The placement chamber for each donor pair and associated test subject changed daily, with the test subjects receiving one conditioning trial in each of the eight chambers over the eight conditioning trials.

Test-subject odor conditioning took place at approximately $0900 \mathrm{~h}$. Eight test subjects, in their home cages, were transferred to a carrying rack and taken to the testing room. Two donor animals were placed consecutively into a particular chamber, where each received the same goal event, either water or an empty cup, 2 min per placement. All eight chambers were used at the same time, so that 4 donors were in chambers with water, and 4 donors in chambers with empty cups. After removal of the second donors, the used drinking cups were replaced with clean cups containing $30 \mathrm{ml}$ of water. As much as possible, the placement chambers were kept closed to minimize odor loss.

Next, 8 test subjects, representing all experimental and control groups, were placed for $10 \mathrm{~min}$ into specific experimental chambers, depending on their group designation. Immediately upon removal, the experimental subjects given the CS+ odor received a $\mathrm{LiCl}$ injection in the testing room, and were then returned to their home cages. Drug injections given during the course of the experiment were administered intraperitoneally. Drug dosage was $12 \mathrm{ml} / \mathrm{kg}$ body weight of a $0.15 \mathrm{M}$ lithium chloride solution. The subjects removed from the CS- odor were returned directly to their home cages. All home cages were then returned to the vivarium.

The control subjects were similarly treated; however, the $\mathrm{LiCl}$ injections occurred at least $4 \mathrm{~h}$ after the last placement in the testing room. That is, like the test subjects, the control subjects also had one of the odors designated as CS + . Half of each control group, 
$\mathrm{CN}$ and $\mathrm{CR}$, had $\mathrm{R}$ odor as the CS+, and half had $\mathrm{N}$ odor. On all 8 days of conditioning, the control animals were returned to the vivarium immediately upon removal from an odor-containing chamber. On days when the odor was $\mathrm{CS}+$, the control subjects were returned to the testing room $4 \mathrm{~h}$ after being removed, and were given a $\mathrm{LiCl}$ injection.

After a squad of test subjects finished a trial in the chambers, the chambers were raised, and the white paper that covered their floor was removed and discarded. In addition, the walls and roof of the chambers were swabbed with a wet cloth. Four hours after placement conditioning, all test subjects received their daily water maintenance in their home cages.

Odor conditioning testing. Five days after Day 8 of conditioning, testing began. Testing consisted of placing a test subject into a placement chamber containing the test odor for a 10-min period, and then measuring the amount of water consumed from a cup containing $30 \mathrm{ml}$ of water. Odors were generated in the usual way by the donor animals. The test animals were given one test per day for 4 days.

\section{RESULTS}

\section{Donor Runway Speeds}

Donor traversal times over the last three sessions of runway conditioning were recorded to assess whether the donors were emitting differential odors on $\mathrm{R}$ and $\mathrm{N}$ trials. Prior work has shown that, if the odor concentration is sufficient, rats will display differential traversal speeds in the goal area. Furthermore, giving successive animals the same treatment assures the buildup of sufficient odor. To assess odor production, we examined traversal speeds for the rats fifth or later in the order of successive animals.
Times to traverse the goal section were converted to goal speeds $(\mathrm{cm} / \mathrm{sec})$. The unit of statistical analysis was mean goal speed for an animal over two successive trials with the same goal event. Across both squads, mean goal speed to $\mathrm{R}(M=42.5)$ was greater than mean goal speed to $\mathrm{N}$ $(M=28.73)$, clearly indicating differential responding and, by inference, differential odor production. A withinsubjects analysis of variance (ANOVA), with six trial blocks and $\mathrm{R}$ versus $\mathrm{N}$ as factors, revealed a significant effect of $\mathrm{R}$ versus $\mathrm{N}[F(1,14)=12.43, p=.01]$. No other effects were significant.

\section{Odor Conditioning}

Figure 1 displays water consumption across test trials for the test animals. An ANOVA, with groups and days as factors, found a significant groups effect $[F(5,54)=$ $5.21, p=.0006]$, but neither the days effect $[F(3,162)$ $=1.99, p>.05]$ nor the groups $\times$ days interaction effect $[F(5,162)=1.06, p>.05]$ was significant. Subsequent $\boldsymbol{t}$ tests indicated that Groups NN and RR differed significantly $(p<.05)$ from both control groups [Group CR: $t(54)=3.87, t(54)=2.69$; and Group $C N$ : $t(54)=4.28, t(54)=3.09$, respectively]. In addition, Group NN differed from Groups RN $[t(54)=2.69$, $p<.05]$ and NR $[t(54)=2.37, p<.05]$, but Group RR did not $[t(54)=1.58, t(54)=1.19$, all $p s>.05]$. This pattern of results may be interpreted most simply, perhaps, by noting that there were no significant differences between pairs of groups given similar treatments. That is, the two CS+ groups (NN and RR) were

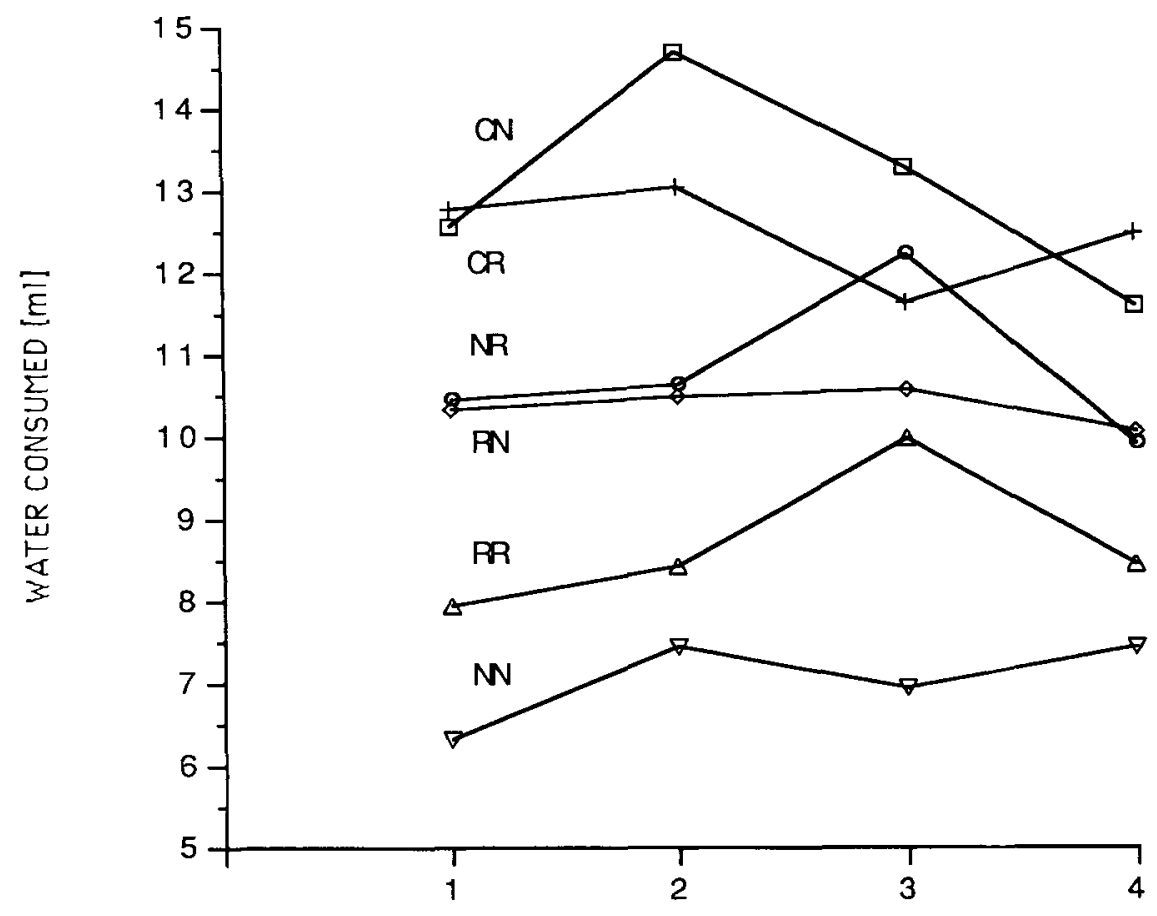

TEST TRIALS

Figure 1. Mean water consumption of the six conditioning groups over the 4 days of testing. 
not significantly different $[t(54)=1.19, p>.05]$, nor were the two CS- groups (NR and RN) $[t(54)=.31$, $p>.05]$, or the two controls (CN and CR) $[t(54)=.40$, $p>.05$ ]. Indeed, only in the case of the CS+ groups was the difference in consumption at all substantial. Combining these similarly treated pairs results in a simple description of the data: Consumption in the presence of the CS- odor was significantly less than that of the controls $[t(54)=3.09, p<.05]$, and consumption in the presence of the CS+ odor was less than it was in the presence of the CS- odor $[t(54)=2.37, p<.05]$.

Since all treatments involved $\mathrm{LiCl}$ injections, it cannot be determined with certainty whether the control groups, $\mathrm{CR}$ and $\mathrm{CN}$, displayed any suppression because of the injection. However, any suppression that the injection might have caused did not appear under control of odor. Since the subjects in the control condition always received $\mathrm{LiCl}$ injections $4 \mathrm{~h}$ after exposure to a particular odor, either $\mathrm{R}$ or $\mathrm{N}$, we could assess whether any association was formed between odor and illness under these conditions. An ANOVA of consumption for the control subjects, with injection-day odor and test-day odor as factors, found no effect that even approached conventional significance levels. Therefore, the training procedure for the controls accomplished its mission: The time gap between odor and injection was long enough to provide a baseline for which the injection appeared functionally independent of odor.

\section{DISCUSSION}

Numerous studies have found that the pattern of goal speeds exhibited by our donor subjects represents a discrimination in which differential odors provide the discriminative stimuli. Other possible cues, such as droppings from the reinforcer itself or differential urine, cannot account for the differential reactions (Davis \& Weaver, 1981; McNeese, 1975; Travis-Neideffer, 1981). Furthermore, Taylor and Ludvigson (1987) have directly demonstrated that $\mathbf{R}$ and $\mathbf{N}$ goal events result in volatile compounds that support differential responding. Therefore, the inference seems secure that our donor animals emitted differential odors in the presence of $\mathrm{R}$ and $\mathrm{N}$ events prior to the odor aversion conditioning phase of the experiment. Equally compelling is the inference that these odors were acting as differential conditioned stimuli controlling aversion in the test subjects. Care was taken to keep all factors constant from one test trial to the next, except for the particular goal event received by the donors. Pertinent to this, the donors were the same animals on $\mathrm{CS}+$ and CS - trials for any given test animal, thus ruling out differences in odors characteristic of different animals. In fact, it is interesting, in this regard, to contrast the present effect with the poisoned-partner effect (PPE) (e.g., Gemberling, 1984; Revusky, Coombes, \& Pohl, 1981, 1982). Unlike in PPE experiments, in the present study, the test subjects never had contact with the donor "partner," nor was the donor made ill at any time. Therefore, although the PPE effect could result from a variety of cues, the present effect seems more certainly to arise from differential odors from the $\mathrm{R}$ and $\mathrm{N}$ goal events.

Given the presence of differential odors, our aversive conditioning data indicate that both $\mathrm{R}$ and $\mathrm{N}$ odors can be aversively conditioned: drinking in Groups NN and RR was markedly suppressed. Furthermore, the fact that suppression was greater for CS+ odors than it was for CS- odors indicates that rats readily discriminate between $\mathrm{R}$ and $\mathrm{N}$ odors if one has been aversively conditioned.

Although consumption was greater in the presence of the CS- odor than it was in the presence of the CS+ odor, it was not up to the controls' level of consumption. Two explanations can be offered for this difference. Stimulus generalization from CS + to CS- odors might have held down water consumption in groups tested with CS- . Alternatively, since the CS- odors were presented in the environment that had immediately preceded aversive conditioning, the environment also might have gained a measure of aversiveness (P. J. Best, M. R. Best, \& Mickley, 1973; M. R. Best, Brown, \& Sowell, 1984). That is, the experimental animals might not have been affected solely by the odor, but also by the presence of an aversive environment.

A major interest of the present study was whether $\mathrm{N}$ odor would be a stronger conditioned suppressor of consumption than would R odor, because of its presumed preexperimental "meaning," a kind of hypothetical unconditioned reaction or representation. If $\mathrm{N}$ odor conveyed a rather general "aversive" meaning, then it presumably would be easy to associate with aversive events via aversive conditioning, whereas it would be much more difficult to aversively condition $\mathrm{R}$ odor, because of its general "appetitive" meaning. However, the present data provide only a hint that $\mathbf{N}$ odor is more readily aversively conditioned. The greater suppression of water consumption did indeed occur in the group that had $\mathbf{N}$ odor, rather than $\mathrm{R}$ odor, as a CS + , but it was not significantly greater. Since there seems to have been no artificial floor to the response measure preventing even greater suppression in this group, the hypothesis of general meaning is not favored. It remains possible, of course, that these odors nevertheless possess such general meanings, but that other techniques are required to reveal them. ${ }^{1}$

Although other studies point to some innate meanings associated with these odors, and they may well be specific rather than general, perhaps the overriding conclusion from the various pertinent studies is that the odors function primarily as salient stimuli-stimuli of high associability but low unconditioned meaning. With this interpretation, $\mathrm{N}$ and $\mathrm{R}$ odors are innately tied only weakly to specific events or classes of events, and either odor can be associated with either congruent or incongruent events with little difficulty. Experiments on constraints on association due to innate meaning of these odors have found constraints to be weak and short-lived (Eslinger \& Ludvigson, 1980; Travis-Neideffer, 1981). In fact, these studies have shown that after $\mathrm{R}$ and $\mathrm{N}$ odors have func- 
tioned as discriminative stimuli for either congruent or incongruent goal events (with the initial congruent association the easier), the discriminations are readily reversible. In sum, then, the data indicate that these odors exercise only weak constraint on associability, and they suggest that the odors can function as signals for a variety of events. A form of communication is suggested that resembles, at least superficially, our own: Odors constitute signals that can readily acquire meaning, rather than just emissions that trigger a stereotyped response.

Consistent with this view, but stressing the role of the reinforcer in the animal's processing of the stimuli presented, it may be that aversive conditioning via toxicosis is such an overwhelming experience that the animal tends to ignore any innate meaning of the odor in favor of determing a reliable predictor for the illness. That is, innate meaning-even a general one-may be important only when present reinforcement is not overly potent.

Finally, the methodological significance of the study should be noted. Naturally produced odors were aversively conditioned with ease using a differential conditioning procedure. This technique should prove useful for investigating a variety of questions.

\section{REFERENCES}

Best, M. R., Brown, E. R., \& Sowell, M. K. (1984). Taste-mediated potentiation of noningestional stimuli in rats. Learning \& Motivation, $15,244-258$.

Best, P. J., Best, M. R., Mickley, G. A. (1973). Conditioned aversion to distinct environmental stimuli resulting from gastrointestinal distress. Journal of Comparative \& Physiological Psychology, 85, 250-257.

Davis, S. F., \& Weaver, M. S. (1981). Odor-mediated patterned responding as a function of delay of reinforcement but not rewardmagnitude contrast. Bulletin of the Psychonomic Society, 18, 331-337.

Domjan, M. (1973). Role of ingestion in odor-toxicosis learning in the rat. Journal of Comparative \& Physiological Psychology, 84, 507-521.

EsLinger, P. J., \& Ludvigson, H. W. (1980). Are there constraints on learned responses to odors from rewarded and nonrewarded rats? Animal Learning \& Behavior, 8, 452-456.

GemberLing, G. A. (1984). Ingestion of a novel flavor before exposure to pups injected with lithium chloride produces a taste aversion in mother rats (Rattus norvegicus). Journal of Comparative Psychology, 98, 285-301.

LoRden, J. F., Kenfield, M., \& Braun, J. J. (1970). Response suppression to odors paired with toxicosis. Learning \& Motivation, 1 , $391-400$.

Ludvigson, H. W. (1969). Runway behavior of the rat as a function of intersubject reward contingencies and constancy of daily reward schedule. Psychonomic Science, 15, 41-43.

Ludvigson, H. W., Mathis, D. A., \& Choquette, K. A. (1985). Different odors in rats from large and small rewards. Animal Learning \& Behavior, 13, 315-320.
Ludvigson, H. W., Sytsma, D. (1967). The sweet smell of suc cess: Apparent double alternation in the rat. Psychonomic Science, 9, 283-284

McHose, J. H., \& Ludvigson, H. W. (1966). Differential conditioning with nondifferential reinforcement. Psychonomic Science, 6, 485-486.

MCNEESE, R. R. (1975). The effects of gonadectomy on the production, avoidance and discrimination of frustration odor. Unpublished master's thesis, Texas Christian University, Fort Worth, TX.

Mellgren, R. L., Fouts, R. S., \& Martin, J. W. (1973). Approach and escape to conspecific odors of reward and nonreward. Animal Learning \& Behavior, 1, 129-132.

Morrison, R. R., \& LUDVIGSON, H. W. (1970). Discrimination by rats of conspecific odors of reward and nonreward. Science, 167, 904-905.

Panhuber, H. (1982). Effect of odor quality and intensity on conditioned odor aversion learning in the rat. Physiology \& Behavior, 28, 149-154.

PRYTUla, R. E., \& DAvis, S. F. (1974). Runway performance as a function of positively and negatively correlated olfactory cues. Psychological Reports, 35, 735-740.

Prytula, R. E., Davis, S. F., \& FanNing, J. J. (1981). The acquisition of a running response as a function of odor buildup, squad rotation, and introduction of naive subjects. Animal Learning \& Behavior, 9, 556-560.

Revusky, S., Coоmbes, S., \& PoHL, R. W. (1981). Failure of albino guinea pigs to exhibit Lavin's poisoned partner effect. Behavioral \& Neural Biology, 32, $111-113$.

Revusky, S., CoOmbes, S., \& PoHL, R. W. (1982). US preexposure: Effects on flavor aversions produced by pairing a poisoned partner with ingestion. Animal Learning \& Behavior, 10, 83-90.

TAUXULIS, H. K. (1974). Odor aversions produced over long CS-US delays. Behavioral Biology, 10, 505-510.

TAYLOR, R. D., \& LUDVigSON, H. W. (1980). Selective removal of reward and nonreward odors to assess their control of patterned responding in rats. Bulletin of the Psychonomic Society, 16, 101-104.

TAYLOR, R. D., \& LUDVIGSON, H. W. (1987). Airborne differences in odors emitted by Rattus norvegicus in response to reward and nonreward. Journal of Chemical Ecology, 13, 1147-1161.

Travis-NeIDEFFER, M. N. (1981). Overshadowing/generalization vs. preexperimental bias in utilization of motivation-specific odors in rats. Unpublished doctoral dissertation, Texas Christian University, Fort Worth, TX.

VOORHEES, J. W., \& ReMLey, N. R. (1981). Mitral cell responses to the odor of reward and nonreward. Physiological Psychology, 9, 164-170.

\section{NOTE}

1. This was not the first time that we could find no evidence for a general meaning. A prior pilot study with only 5 subjects per group found a suggestive nonsignificant difference similar to that of the present work, but even combining probabilities from the two studies did not yield a probability value that approached conventional levels of significance.

(Manuscript received April 11, 1988; revision accepted for publication August 19, 1988.) 